\title{
A long term spectroscopic and photometric study of the old nova HR Delphini ${ }^{\star}$
}

\author{
M. Friedjung ${ }^{1}$, M. Dennefeld ${ }^{1, \star \star}$, and I. Voloshina ${ }^{2, \star \star \star}$ \\ 1 Institut d'Astrophysique de Paris -UMR 7095, CNRS/Université Pierre et Marie Curie, 98bis boulevard Arago, 75014 Paris, France \\ e-mail: fried@iap.fr \\ 2 Sternberg Astronomical Institute, 119992 Moscow, Russia
}

Received 21 December 2009 / Accepted 17 June 2010

\section{ABSTRACT}

Context. The Nova HR Del, discovered in 1967, was found to be exceptionally bright in the optical and UV during the whole lifetime of the IUE satellite (ending in 1996) and appears to still be extremely luminous today. The reason for this continuing activity is not clear, but continuing weak thermonuclear burning might be involved.

Aims. We therefore need to better understand the processes at play in HR Del.

Methods. HR Del was thus monitored over several years, both in broad band photometry and spectroscopically in the H $\alpha$ spectral region.

Results. The profile of the $\mathrm{H} \alpha$ line shows two components: a narrow, central component and broader wings. The former is most easily understood as the result of an accretion disk whose geometry might lead it to partly occult certain regions of itself. That component shows something like an $\mathrm{S}$ wave with an orbital phase dependence, suggesting that it could be due to a spot bright in $\mathrm{H} \alpha$. The wide component must come from another region, with a probably non-negligible contribution from the material ejected during the 1967 outburst. Non-orbital variations of the $\mathrm{H} \alpha$ equivalent width were found both on long and short time scales. Similar variations were found in the photometry, showing a component with a clear dependence on the orbital phase, however with no obvious relation with the $\mathrm{H} \alpha$ variations.

Conclusions. The orbital part of the photometric variations can be explained by irradiation of the companion, while the properties of $\mathrm{H} \alpha$ are explicable by the presence of an accretion disk and a spot bright in $\mathrm{H} \alpha$.

Key words. novae, cataclysmic variables - circumstellar matter - stars: individual: HR Del - stars: individual: V723 Cas stars: individual: V603 Aql

\section{Introduction}

HR Delphini (or Nova Del 1967) was a bright classical nova with, however, unusual properties. It was discovered on July 8 1967 by Alcock (see Candy 1967), when it had brightened to a magnitude near 5.5 from a pre-outburst magnitude of about 12 at the beginning of June. After having remained 5 months near this magnitude, it brightened again to a short peak at $m_{v}$ of 3.5 in December 1967, which probably corresponds to what is considered to be the maximum for classical novae. This was followed by an extremely slow irregular decline, as can be seen for instance in Sanyal (1974). During the stage before the brightest peak, the velocity of the main absorption component continuously decreased, unlike the usually observed behaviour of velocities in classical novae after maximum. This reinforces the idea that the short peak in December 1967 was indeed the "real" maximum. The unusual behaviour of this nova in such an exceptionally long pre-maximum stage might, according to

* A journal of the CCD photometric observations is presented in Table 1. Observing data for individual spectra are presented in Tables 2 and 3. All these tables are only available in electronic form at http: //www . aanda. org

$\star \star$ Visiting Astronomer, Observatoire de Haute-Provence, CNRS, France.

$\star \star \star$ Visiting Astronomer, Crimean station of the Sternberg Astronomical Observatory.
Friedjung (1992), be explainable by the presence of a wind, which was optically thin in the continuum, and with a decreasing velocity during that pre-maximum stage, unlike the velocities of the optically thick winds normally seen in classical novae after maximum. HR Del may, indeed, only have marginally satisfied the conditions needed for thermonuclear runaway. This could be due to the very low mass $\left(0.55-0.75 M_{\odot}\right)$ of the white dwarf component of the binary (Selvelli \& Friedjung 2003). A few other classical novae such as V723 Cas (1995) may have a similar nature.

In addition, this nova showed signs of unusual activity both before and long after its outburst (Selvelli \& Friedjung 2003). This can be seen by its exceptional luminosity, compared with that of other old novae both from IUE observations in the UV and from its optical apparent brightness. The time variable $\mathrm{P}$ Cygni profile of the CIV resonance doublet also indicates a very high wind velocity of the post-nova of the order of $5000 \mathrm{~km} \mathrm{~s}^{-1}$. The deduced values of distance of $850 \mathrm{pc}$ and $E(B-V)=0.16$ led to a UV luminosity of $56 L_{\odot}$ and a visual absolute magnitude of $M_{v}=1.85$. The latter value has to be corrected for the inclination of the system: assuming that the radiation came from a gravitationally heated accretion disk (then having an accretion rate of $\mathrm{d} M / \mathrm{d} t=1.4 \times 10^{-7} M_{\odot} \mathrm{yr}^{-1}$ onto a white dwarf with a radius of $0.0125 R_{\odot}$ ), the correction leads to an absolute magnitude of $M_{v}=2.30$. Such observations suggested unusual activity; a possible explanation was continuing very weak thermonuclear 
burning, with the radiation of the white dwarf being reprocessed by an accretion disk (and the companion star), a theoretically possible situation when the white dwarf component's mass is low (Sion \& Starrfield 1994). In this connection we can note that V723 Cas was still an active X-ray source more than 12 years after the outburst (Ness et al. 2008), but in the case of HR Del, only weak X-ray emission was reported at early times when instruments were much less sensitive than now (Hutchings 1980, with $6 \times 10^{31} \mathrm{erg} \mathrm{s}^{-1}$ after correction for our adopted distance), and nothing more recently.

It is possible to obtain a better clue to the luminosity by estimating the ionisation of the nebular remnant by the central source. The remnant of HR Del has been studied in a number of papers, e.g. Harman \& O'Brien (2003). Very recently, Moraes $\&$ Diaz (2009) have studied the clumpy structure of the nebula. Detailed photoionisation modelling suggests that there is a disk shaped ionising source, with a luminosity of $10^{36} \mathrm{erg} \mathrm{s}^{-1}$ and a temperature of $65000 \mathrm{~K}$. The two sides of a disk, emitting like a black body at such a temperature, would then have a radius of $1.3 \times 10^{10} \mathrm{~cm}$ or $0.2 R_{\odot}$. Such a relatively low temperature might explain why the X-ray emission is weak . A total shell mass of $9 \times 10^{-4} M_{\odot}$ was also found by these authors, at least half being contained in the neutral clumps.

In the light of these results, time variations of HR Del need to be followed by ground-based observations, as they could reveal some signs of exceptional activity. It was with this in mind that we observed the region of $\mathrm{H} \alpha$ with a high spectral resolution over several years, since this line could give particularly significant results. Parallel broad band photometric monitoring was also performed. Some preliminary results of this observing campaign have already been presented by Friedjung et al. (2005).

\section{Observations}

\subsection{Photometry}

Photometric observations of HR Del were made in order to detect possible long-term variations, and also to verify the orbital phases for the spectroscopic observations. They were carried out with two different instruments: firstly, the single-channel photoelectric photometer on the 60-cm telescope of the Sternberg Astronomical Institute in Crimea was used from 2002 to 2009, with continuously changing Johnson UBV filters and a $10 \mathrm{~s}$ integration in each filter. The usual method of differential measurements was applied. A local standard was used permanently during observations and calibrated against the well-known standard star SAO 106418 (magnitudes $V=8.59, B=8$ m.62, $U=8$ m 55 ). More than 1600 measurements of HR Del were obtained in 3 bands during the total time of our observations, with usually 2-3 measurements per night. These data were used to determine the long-term behaviour of HR Del, which is shown in Fig. 1. They are accurate to $1 \%$ in the $V$ and the $B$ bands and $2 \%$ in $U$ band.

Secondly, CCD observations of HR Del were obtained with the goal of studying variability on time scales from minutes to days. Several runs of a few nights were made in August and October 2005, during the period of July-October 2006, then during October-November 2008, and finally during three nights in October 2009. Most of these observations were carried out in the $V$ band with the $60 \mathrm{~cm}$ telescope of the Crimean station of the Sternberg Astronomical Institute, using an Apogee 47 CCD detector with $1024 \times 1024,13 \mu \mathrm{m}$ pixels, and a field of view of $6 \times 6$ arcmin. During 2008, in addition to the above instrument used both in the $V$ and $R$ bands, the $38 \mathrm{~cm}$ telescope

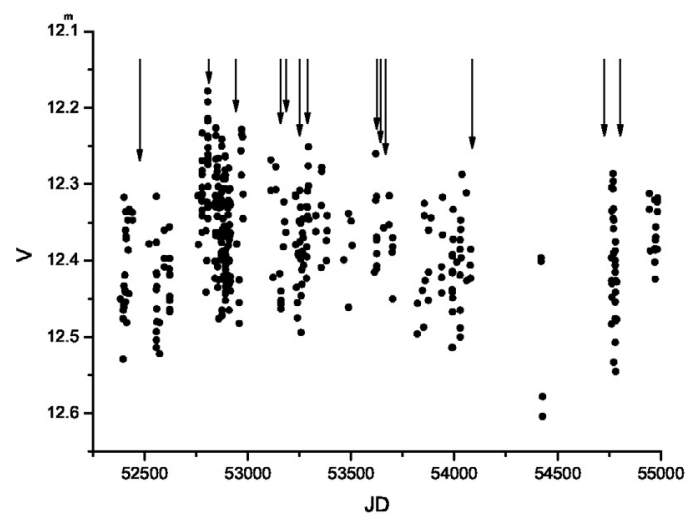

Fig. 1. Overall behaviour of HR Del in the $V$ band during 2002-2008. Photoelectric $V$ magnitudes are shown versus Julian Day. The arrows indicate the epochs of the spectroscopic observations.

of the Crimean Astrophysical Observatory was also used from November 10 to 13 , but only in the $R$ band, with a CCD-ST7 camera $(460 \times 700,9 \mu \mathrm{m}$ pixels $)$. In 2009 , a Pictor MEADE-416 camera was also used on the $50 \mathrm{~cm}$ Sternberg telescope in the $V$ band. All these data were obtained in a $2 \times 2$ binning mode to reduce the read-out time. The duration of individual observational series varied from 2.5 up to $4.5 \mathrm{~h}$. A star close to HR Del in the field was always used as a local standard (a few suitable stars were used, among them the one used for the photoelectric observations), and repeatedly calibrated using well-known comparison stars during a few nights with perfect weather. Two other stars, one with coordinates $\alpha=20^{\mathrm{h}} 42^{\mathrm{m}} 18^{\mathrm{s}}$ and $\delta=+19^{\circ} 08^{\prime} 30^{\prime \prime}$ $V=13 \mathrm{~m} \cdot 660, B=14 \mathrm{~m} \cdot 70, U=15^{\mathrm{m}} \cdot 70$ and another with $\alpha=$ $20^{\mathrm{h}} 42^{\mathrm{m}} 33^{\mathrm{s}}$ and $\delta=+19^{\circ} 06^{\prime} 30^{\prime \prime} V=12^{\mathrm{m}} \cdot 929, B=14^{\mathrm{m}} \cdot 05, U=$ 15 . 03 , were used as control stars. More than 4200 measurements of HR Del were obtained this way in the $V$ band during 2005-2008, covering more than 30 nights, while about 700 datapoints were obtained in $R$ over 9 nights. The CCD measurement errors do not exceed $3 \div 4 \%$ in the $V$ band, and slightly less for the $R$ band.

The details of the observations are presented in Table 1. Examples of daily light curves are presented in Fig. 2. A more detailed description of these observations will be presented in a companion paper (Voloshina et al., in prep.).

\subsection{Spectroscopy}

The spectroscopic monitoring was done at the Observatoire de Haute-Provence (OHP) in France, using the $1.52 \mathrm{~m}$ telescope, equipped with the Aurélie high-resolution spectrograph (Gillet et al. 1994). Light passing through a $3^{\prime \prime}$ circular entrance aperture then encounters a modified Bowen-Walraven image slicer (Walraven 1972), whose 5 slices of $0^{\prime \prime} 6$ each are imaged onto a $2048 \times 1024$ EEV CCD with $13.5 \mu$ m pixels. To gain in sensitivity, all the corresponding lines of the CCD were summed before read-out, producing a single-line spectrum as output. However as a consequence, the possibility of identifying and cleaning cosmic rays hits before final reduction disappears. The spectra were reduced following standard procedures: bias and flat-field corrections, and wavelength calibration using a thorium-argon internal lamp. In view of the narrow spectral range covered and used, no spectral response correction was performed.

Many of the observations (but not all) were done in service modes, and used a $1200 \mathrm{l} / \mathrm{mm}$ grating giving a dispersion of $7.6 \AA$ per $m m$ and a spectral resolution of $0.32 \AA$ ( 0.10 per pixel $)$. 


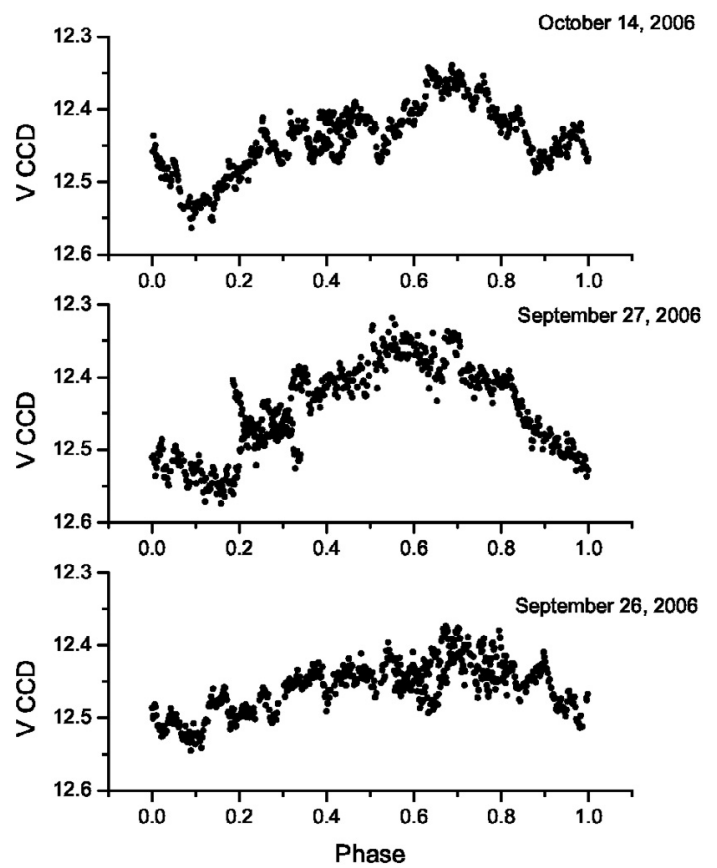

Fig. 2. Examples of CCD observations for different dates: CCD-V magnitudes versus phase.

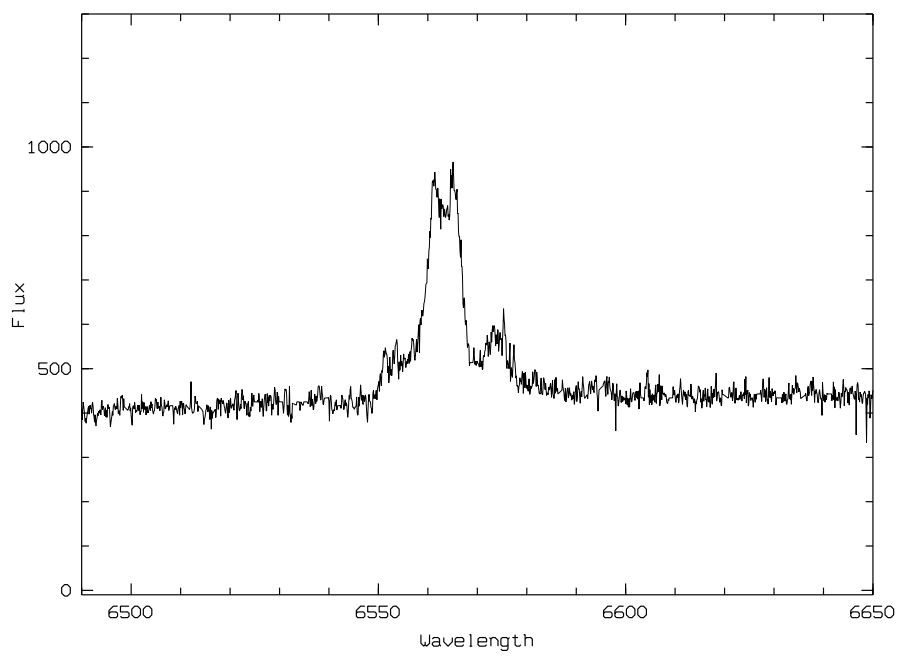

Fig. 3. Example of a high dispersion spectrum, obtained on November 1, 2003 at the OHP. The core and the low-intensity wings of $\mathrm{H} \alpha$ are easily distinguished.

Typical individual exposure times are $30 \mathrm{~min}$, but the sequencing of observations and phase coverage are irregular, a tribute to the service mode operation. The details of observations are given in Tables 2 and 3 . An example of a high-resolution spectrum is given in Fig. 3.

A few low-dispersion spectra were obtained towards the end of the campaign to check the general aspect of the object. We used the OHP 1.93m telescope in December 2007, the Carelec spectrograph and a $300 \mathrm{l} / \mathrm{mm}$ grating giving $1.8 \AA$ per pixel and a $6.5 \AA$ resolution with the $2^{\prime \prime}$ slit used. The same setting was used again in December 2008. In June 2008, we used the IDS at the INT $2.5 \mathrm{~m}$ telescope in La Palma. There, a $630 \mathrm{l} / \mathrm{mm}$ grating gave $0.9 \AA$ per pixel and $2.7 \AA$ resolution with a $1^{\prime \prime} 5$ slit. Those spectra were reduced with standard procedures, including the use of standard star observations to correct for the wavelength-dependent spectral response. In the case of the INT,

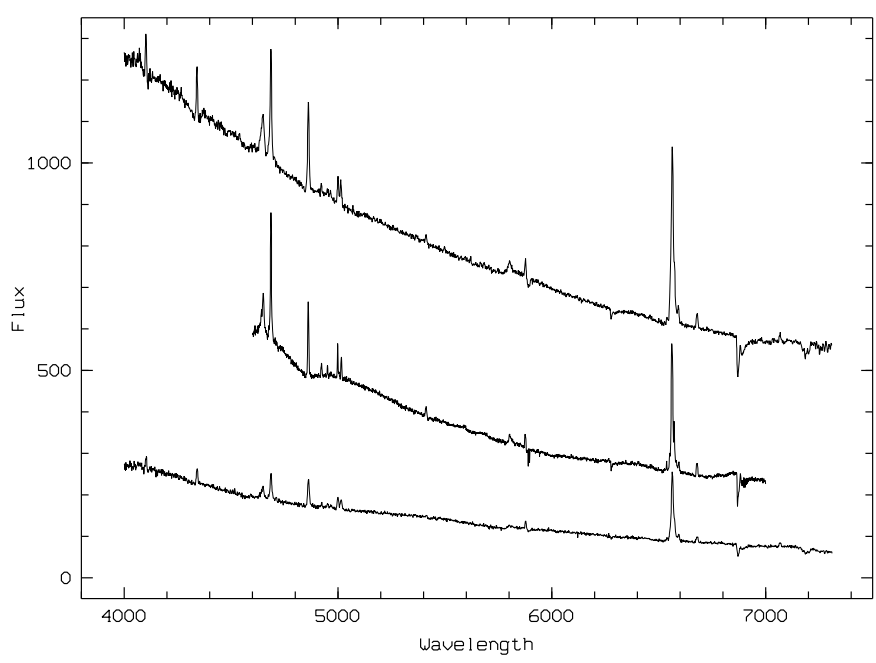

Fig. 4. Low-dispersion spectra. Lower part: OHP, Dec. 2007; Middle: INT, June 2008. Upper part: OHP, Dec. 2008. Small offsets were made between spectra (see text). The units in the ordinates are $10^{-16} \mathrm{erg} / \mathrm{cm}^{2} / \mathrm{s} / \AA$.

however, only the central part of the spectrum can be exploited due to strong vignetting. Summation was done over the full $\mathrm{H} \alpha$ extent along the slit and the spectra are displayed in Fig. 4.

\section{Results and analysis}

\subsection{Analysis of the photometry}

As can be seen from Fig. 2, daily light curves of HR Del show night-to-night variations and aperiodic stochastic variations, that is so-callled flickering. Because they contain long time series of observations at similar epochs, the CCD data were used to search for the orbital period of the light curve. Although covering a longer total timespan we may note that the photo-electric data are too scarcely sampled for that purpose. The analysis was made primarily in the $V$ band, where more data are available, but the $R$ band data were also used to check for possible wavelengthdependent effects. They were analysed with the "Period04" PC code from Lenz \& Breger (2004) and with a programme based on the Deeming (1975) method, in the frequency range $0-50 \mathrm{~d}^{-1}$. The resulting periodogram is displayed in Fig. 5: the most prominent peak is for a period of 0.214164 days, corresponding, within the uncertainties, to the period obtained by Kürster and Barwig from their spectroscopic analysis (0.214165), the other peaks being daily aliases. Our observations folded with this period are shown in Fig. 6. The scatter that remains in this figure comes from night to night variations and possibly also from flickering. Attempts to fold data with periods corresponding to other peaks in the periodogram lead to unacceptable fits, thus confirming the real period. If we approximate this light curve with a sine function (thick, red line), the resulting semiamplitude is $A=0.043 \pm 0.002$.

No other short-term variations were found in these observations besides the orbital ones. To better detect long time-scale variations, such as those found by Bianchini (1987) in some old novae, our data were corrected for orbital variations. There is a suspicion of long-term variations with a possible period of around 1500 days (that is about 4 years), already apparent in Fig. 1, but several more years of observations would be needed to confirm this. 


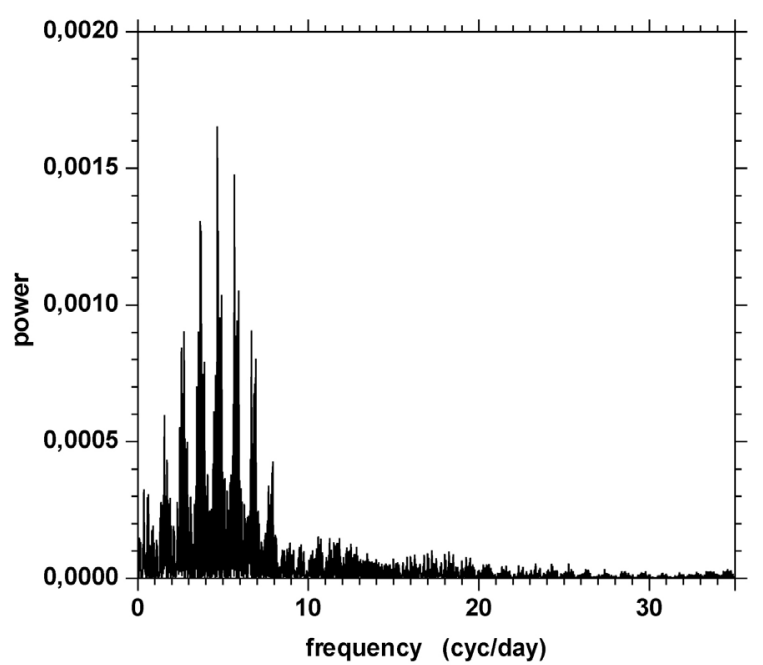

Fig. 5. Power spectrum of the 2008 CCD observations.

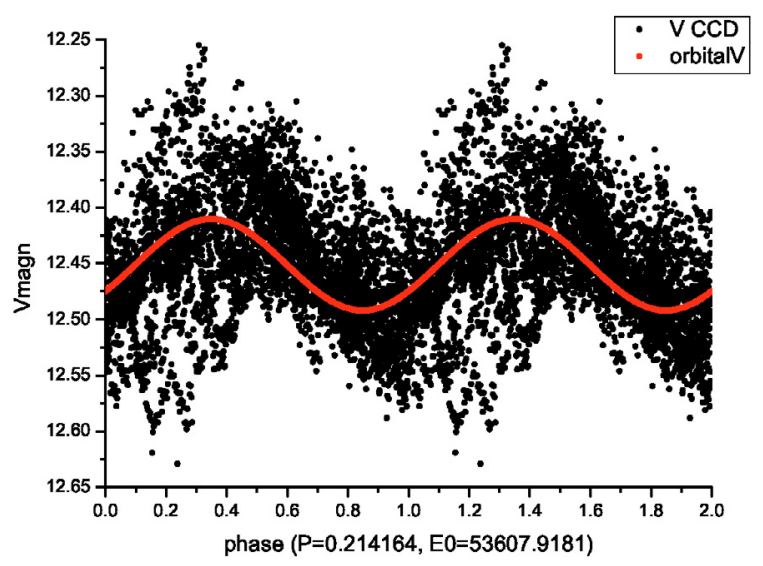

Fig. 6. Light curve of HR Del versus photometric phase, in the $V$ band, from observations obtained during 2005-2006. The averaged orbital sine wave is superposed onto the observed data.

\subsection{Analysis of the $\mathrm{H} \alpha$ spectral region}

The $\mathrm{H} \alpha$ profiles show a well-defined central component, with wider wings of lower intensity (Fig. 3), suggesting formation in different regions. In view of this, measurements were made of both the total equivalent width and the line centre alone.

The time of each spectrum (at mid-integration), the corresponding orbital phase according to the ephemeris of Kürster \& Barwig (1988) and the measured equivalent widths of $\mathrm{H} \alpha$ (both total, and central component only) are given in Tables 2 and 3. Examples of the variation of the equivalent width of the different $\mathrm{H} \alpha$ line components with Julian date and with Kürster and Barwig's orbital phase are shown for two epochs in Figs. 7 and 8, over a time interval not lasting more than a few days. Clear differences are seen between these two examples: on one hand, the average value of the $\mathrm{H} \alpha$ equivalent width is much lower at the first epoch; on the other, the scatter of the variations with phases is more important during the second epoch. Such changes are seen over the whole duration of our observing campaign.

One concern is a possible, time variable, contamination by emission from the ejected nebula in the $\mathrm{H} \alpha$ line due to the central region (plus a much smaller contribution from the fainter, adjacent [N II] emission). To estimate the possible contribution from the nebula, several tests have been made. A detailed map of the nebular emission was obtained by

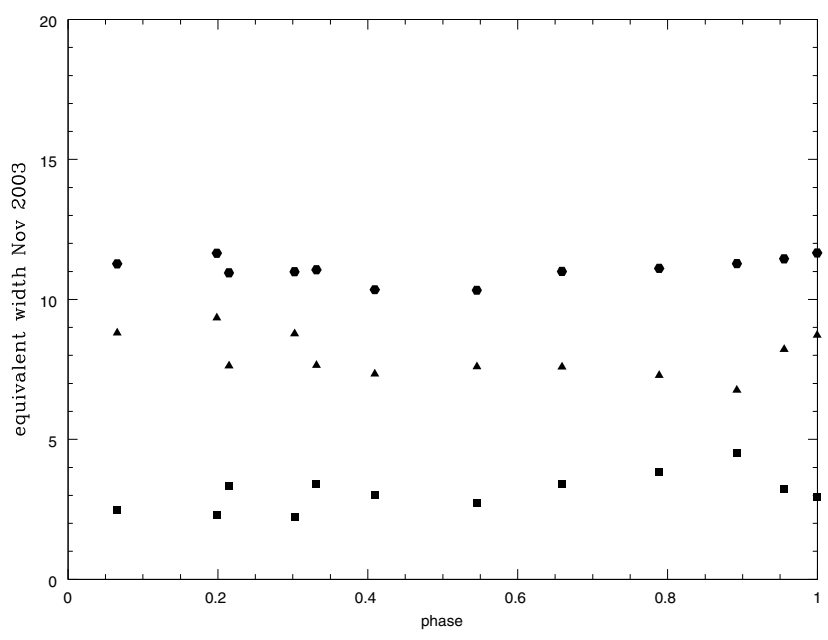

Fig. 7. Equivalent width variation with orbital phase in 2003 November. Filled circles (above) represent the total equivalent width, triangles (middle) the central component and the squares (lower) the wings alone.

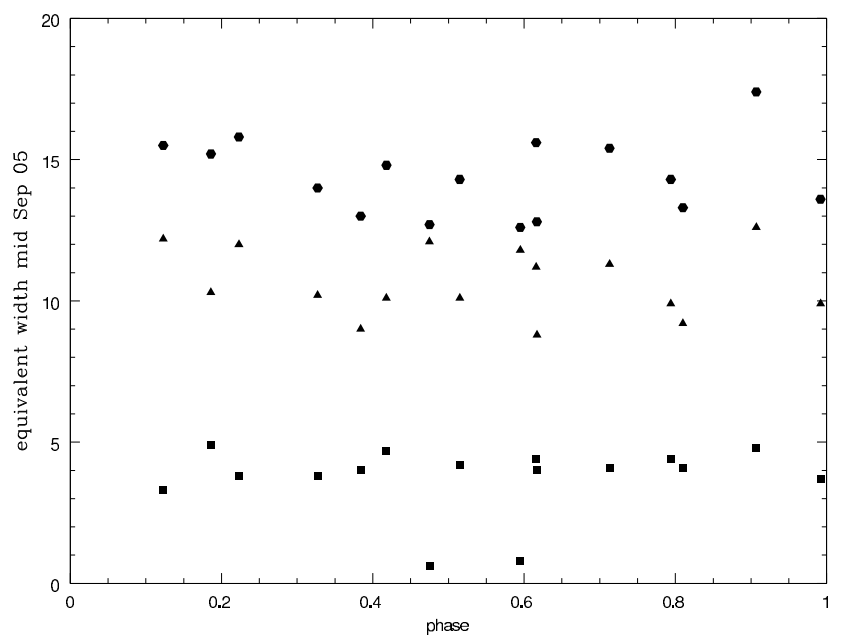

Fig. 8. Equivalent width variation in 2005 mid September. Symbols as above.

Harman \& O'Brien (2003), combining HST images with ground-based, high-resolution spectroscopy. They show that the nebular emission has an elliptical shape, with major and minor axes of roughly $8.5 \times 6.3$ arcsec, with a large $\mathrm{H} \alpha$ contribution coming from a bright rim. These dimensions are much larger than the $3^{\prime \prime}$ entrance aperture, centred on the star, used for our high-dispersion spectroscopy, so that the contribution from the nebula should not be significant in the central $\mathrm{H} \alpha$ component.

The structure of the nebula, as seen in the HST images of Harman \& O'Brien (2003), is however clumpy, and several bright knots, located inside the ellipsoid, had been identified. To measure their possible contribution to the $\mathrm{H} \alpha$ flux from the central object (integrated over a circular aperture, like the $3^{\prime \prime}$ used), it would be sufficient to measure the intensity of those blobs relative to the one of the central star. Unfortunately, the central object is saturated in Harman's data, so we cannot estimate this ratio. We instead used several of our ground-based images, obviously of lesser spatial resolution, but where some of the brightest knots can be identified, and found that the intensity ratio between the brightest knots and the central star is typically 1 to 50 in $\mathrm{H} \alpha$. It would therefore require several knots within the aperture to 


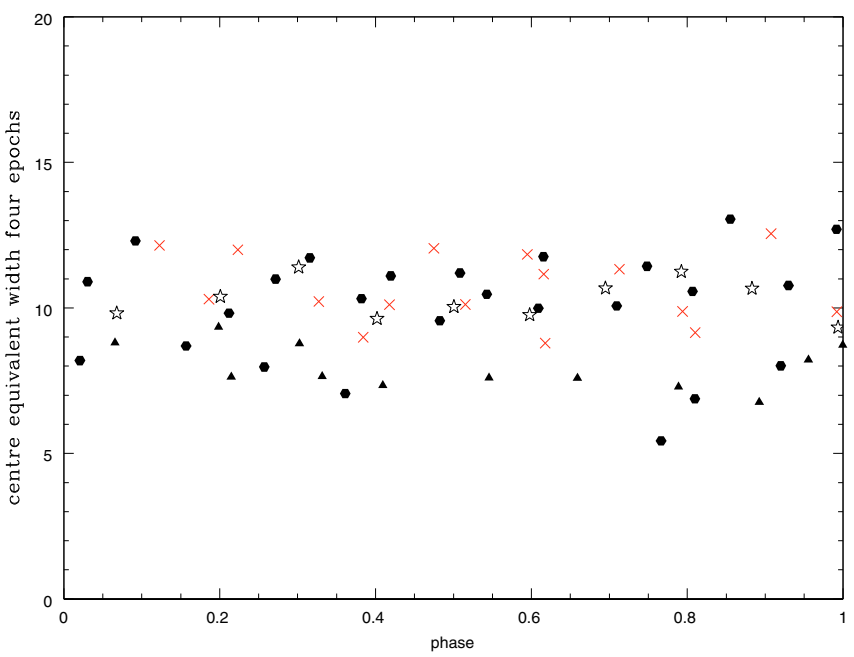

Fig. 9. Equivalent width variation of the central component with phase, for four different observing periods. Triangles are for 2003 November, crosses for 2005 September, open stars for 2005 October and filled polygons for 2008 August.

make a $10 \%$ contribution, and only a few are seen in the whole ellipsoid in the HST map.

We also used the spatial resolution along the slit in our low-dispersion spectra to estimate the change in $\mathrm{H} \alpha$ equivalent width when including more and more outer regions, and found that $10 \%$ was a conservative upper limit for a variable contribution from the nebula (see the discussion below of the lowdispersion spectroscopy). Finally, the measured velocities do not correspond either, at least for the central component, to what is expected from the nebula (see discussion later).

In view of all this, we consider $\mathrm{H} \alpha$ emission line equivalent width variations of more than $10 \%$ as intrinsic, as can be seen in the figures. The low level of the contamination is understandable, following the expansion and fading of the nebula, as even our earliest observations occurred about 35 years after the outburst of the nova.

As the line wings are always faint, the value of the total $\mathrm{H} \alpha$ equivalent width is dominated by the value of the narrow central component, so the variations in these two components are similar; however, it is not clear to what extent any of the orbital phase variations are significant. In some cases, like 2003 November (Fig. 7) or 2005 October, the variations are small: for instance, on November 1-2, 2003, the ratio between the highest and lowest values of equivalent width is only 1.13 . This can give a limit to deviations from circular symmetry at that time. In other cases, e.g. 2005 September (Fig. 8), or 2008 August, the variations, and the scatter are much larger. As more observing points are available in those longer series, these larger variations are probably indicative of rapid variations from night to night.

There are, however, significant differences in average equivalent width from one epoch to the next, as is illustrated in Fig. 9 for the central component: lower values are found for the 2003 epoch, with comparatively stable values over different phases, while higher values are found in September 2005, and a much larger change with phase in 2008.

Quite large, longer time-scale variations of the total and line centre equivalent widths are seen (e.g. Fig. 13, for the central component). The values are clearly lower in 2003 and 2004 than earlier or later. Such variations would be hard to explain by contamination from varying contributions of flux from the ejected nebula. For the wings, no clear systematic variation with time of

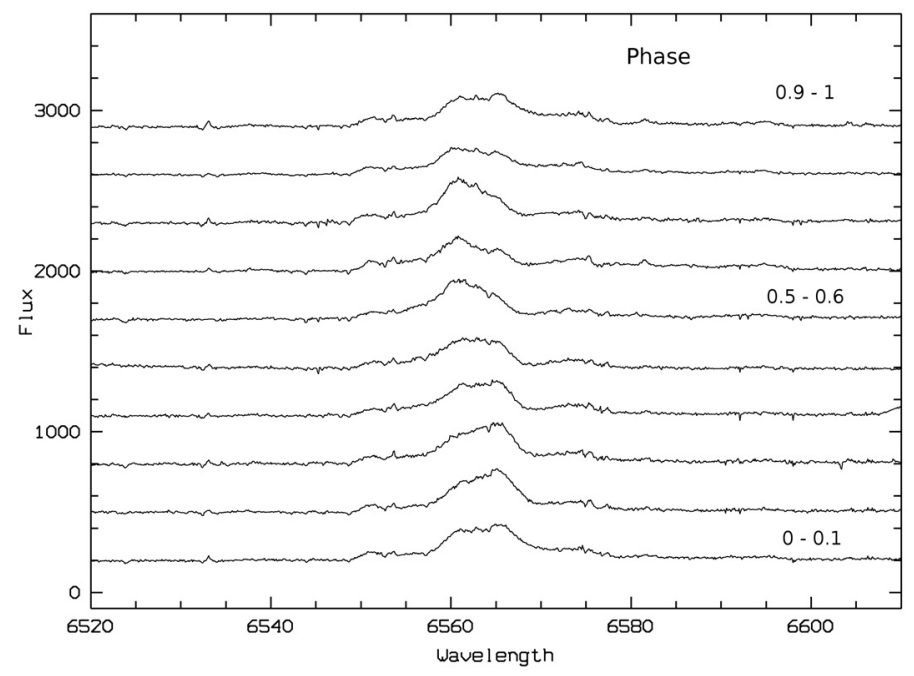

Fig. 10. Series of profiles obtained during 2002-2004, averaged in ten bins of 0.1 in phases. The phases are going from 0 at the bottom (bin 0 to 0.1 ) to 1 at the top (bin 0.9 to 1 .

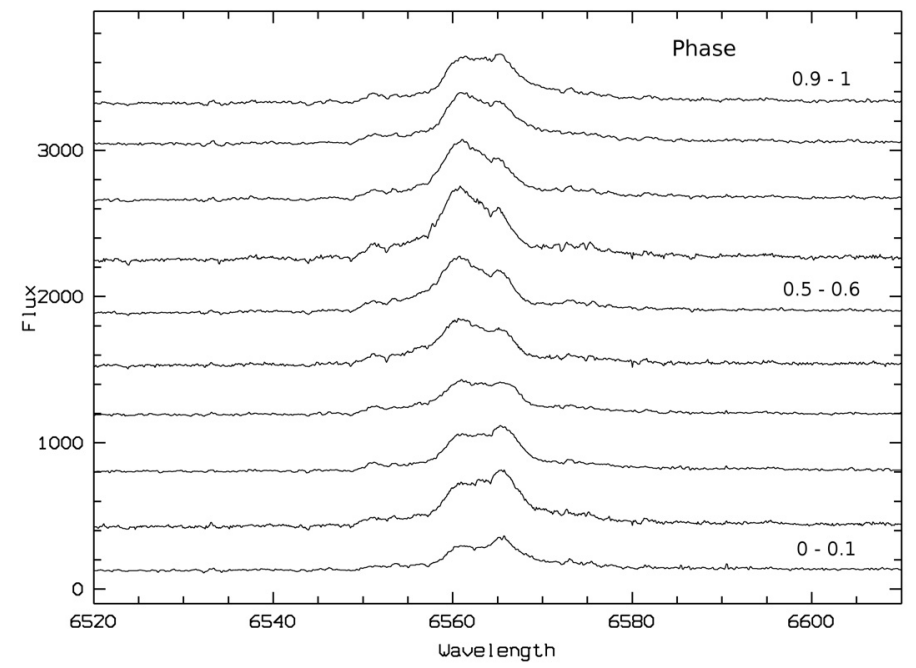

Fig. 11. Series of averaged profiles obtained in 2005 (binning and phases as above).

their equivalent width can be established, as the scatter is large, but changes in their profiles and/or terminal velocity are definitely seen.

The central component of the $\mathrm{H} \alpha$ profile is assymmetric and varies with orbital phase, suggesting something like the well known $\mathrm{S}$ wave of cataclysmic variables. Comparison of profiles from successive spectra, obtained at some observational epochs when fairly long time series of observations could be made, reveals this effect. Three examples are displayed, from the beginning, middle and end of the campaign, in Figs. 10-12, respectively, where series of spectra from the same epoch have been binned over 0.1 intervals of phases. They show the permanency and the stability of the phenomenon. The detailed profiles may not look exactly the same, for a given bin, at each of the three epochs displayed, but these small differences are due to the averaging process; although the total number of observed spectra was quite large, the number of spectra available for a given bin at a given epoch is small, varying between 1 and 5 , and furthermore the phase distribution within a given bin is quite inhomogeneous, so that the "average" aspect can be strongly influenced by the quality or phase of a single spectrum in the average. 


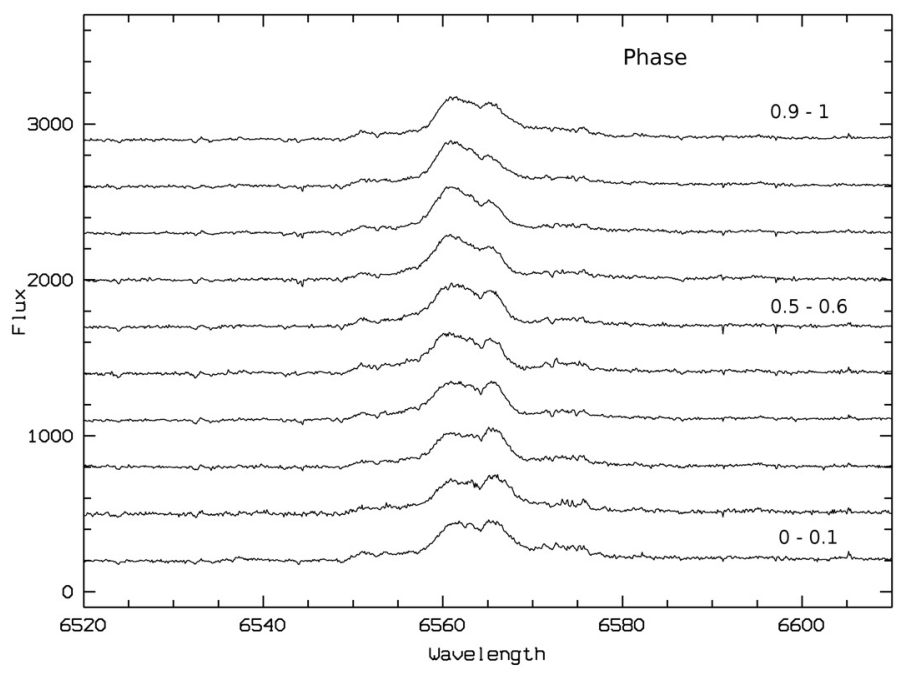

Fig. 12. Series of averaged profiles obtained in 2008 (binning and phases as above).

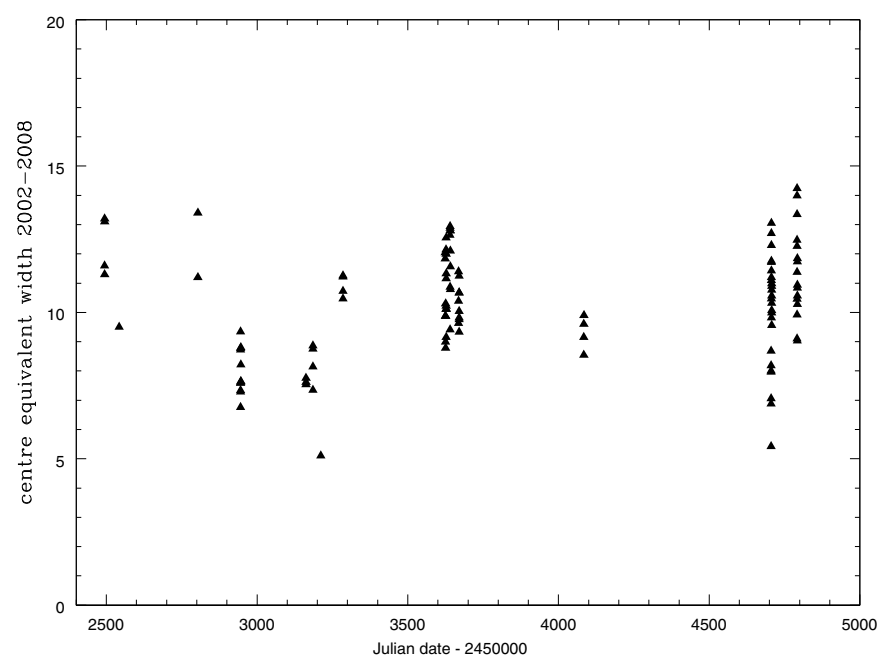

Fig. 13. Long time-scale equivalent width variation of only the central component.

Nevertheless, it is clear that the main features, like the phase of the maximum blue or red peak in the central component are quite clear and reproducible. This also confirms that the spectroscopically determined period of Kürster \& Barwig (1988) is the most appropriate for our analysis.

\subsection{Low-dispersion spectroscopy}

The three spectra shown in Fig. 4 are rather similar: the lower one was obtained at the OHP on 2007 Dec. 10 through thin clouds (exposure of $10 \mathrm{mn}$ ), while the upper one was obtained at the OHP on 2008 Dec. 7, in good conditions (exposure of $5 \mathrm{mn}$ ); the middle spectrum is from the INT on June 25, 2008 (exposure of $200 \mathrm{~s}$ ). The extremities of this INT spectrum (bluewards of $\sim 5000 \AA$ and redwards of $\sim 7000 \AA$ ) are not usable for determining the fluxes because of the onset of a strong vignetting in the camera, which is difficult to correct for in the reduction (the bump seen in the continuum around $5000 \AA$ is also artificial). All three spectra are calibrated, and the differences in ordinates mainly reflect the difference in atmospheric transparency at the moment of the observations (except that the upper spectrum has been offset upwards by 300 units for clarity).
The emission features detected comprise the Balmer lines of hydrogen $(\mathrm{H} \alpha, \mathrm{H} \beta, \mathrm{H} \gamma, \mathrm{H} \delta)$, lines of neutral helium $(7065,6678$, 5876, 5015, $4921 \AA$ ), the HeII lines at 5411 and $4686 \AA$, as well as the broad CIII/NIII $\lambda \lambda 4640$ and CIV $\lambda \lambda 5805 \AA$ features. The slightly higher spectral resolution of the INT (middle) spectrum clearly shows [N II] 6548, $6584 \AA$ A components contributing to $\mathrm{H} \alpha$. There is no indication of a significant variation, from one spectrum to another, of the high-excitation lines, which could be related to the variability of the exciting source.

When comparing with the latest available spectrum in the literature, obtained in 1990 (see Ringwald et al. 1996), we can note that the high excitation lines (HeII, CIV) are still strong, but that the [OIII] $\lambda \lambda 4959,5007 \AA$ lines, present in addition to the classical accretion disk lines, are much weaker now, as expected because of the expansion of the nebula. In practice, we detect only one, faint [OIII] component, blueshifted by $500 \mathrm{~km} \mathrm{~s}^{-1}$, which may correspond to one of the nebular spots detected by Harman \& O'Brien (2003).

The equivalent width of $\mathrm{H} \alpha$ has also been measured on our low-dispersion spectra; the comparison of the long-slit, integrated values with the high spectral resolution values found with the 3 " circular aperture, can indicate the contribution of the nebula to the measurements. When integrating all emission along the slit (extension about 13") in our 2008 December spectrum, for instance, we find a total equivalent width of $\mathrm{H} \alpha$ of $-20 \AA$, which is larger than any value found on high-dispersion spectra at the same epoch. But if we restrict the integration in the longslit spectrum to only the central $3^{\prime \prime}$, we only find $-16 \AA$, in good agreement with the high-resolution value. There is therefore at most a $20 \%$ contribution of something else to the central value extending to large radii from the central object.

The INT spectrum, obtained with a 1."5 slit in good seeing conditions $\left(\sim 1^{\prime \prime} .0\right)$ similarly yields an equivalent width for the central core of the $\mathrm{H} \alpha$ line of $-13.4 \AA$, in excellent agreement with the high-resolution spectra core values. We can thus safely consider that most of the $\mathrm{H} \alpha$ emission comes from the very central parts and that $10 \%$ is a conservative upper limit for the possible contribution of the nebula (or the nebular blobs) to the equivalent width of the central component of $\mathrm{H} \alpha$ in the highresolution spectra.

\section{Discussion}

The first point to emphasise is that the real spectroscopic phases of HR Del are uncertain. Kürster \& Barwig (1988) estimate an error of $5 \times 10^{-6}$ days for a spectroscopic period of 0.214165 days. Their observations were obtained in 1978-1980, of the order of 9000 days before our observations, so the phase uncertainty since then could be not far from one orbital period. We can thus only be reasonably certain about the relative spectroscopic phases at each epoch of our observations, but we can seek information about the real phases using another approach. It should be kept in mind that Kürster \& Barwig (1988) used a convention for spectroscopic binaries, with zero phase at maximum radial velocity, which is different from the one generally used for cataclysmic binaries. The photometric data used the convention for eclipsing variables, with zero phase at the minimum of the light curve (as in Fig. 6, the zero point being given in the figure caption). In fact, by comparing their zero point with our photometric zero point, we find that the Kürster and Barwig phase (NOT the true spectroscopic phase) corresponding to our photometric zero point, zero phase (as in our Fig. 6) is 0.42 . 
A particularly striking feature of our spectra is that the $\mathrm{H} \alpha$ profile seems to be a sum of the already mentioned central and wing components. It is hard to make a comparison with the lower resolution spectra of Kürster \& Barwig (1988) with resolutions of 1 and $2 \AA$, taken from 1978 to 1980 , because those spectra still had serious interpretation problems due to contamination by nebular emission.

We can compare the radial velocities corresponding to the widths of the different components of our high-resolution $\mathrm{H} \alpha$ profiles with the velocities of the emission produced by the expanding nebula. Harman \& O'Brien (2003) find a maximum of about $600 \mathrm{~km} \mathrm{~s}^{-1}$ for the radial velocity of major nebular $\mathrm{H} \alpha$ emission, such a low velocity being expected for this slow nova. Moraes \& Diaz (2009) also find that the highest radial velocity of $\mathrm{H} \alpha$ emitting material is associated with the polar caps, and has a radial velocity of $630 \mathrm{~km} \mathrm{~s}^{-1}$. If a nebular contribution were important, our $\mathrm{H} \alpha$ profile would be expected to show a component with about twice this width in velocity units and yet the dominant central component is much narrower. The limits in radial velocity of the line centre and line wing components are easily measured in our spectra, as seen for instance in Fig. 3: the violet edges are at about -250 and $-650 \mathrm{~km} \mathrm{~s}^{-1}$ for the central component and the blue wing, respectively and similar values are found on the red side (although there they could be slightly modified owing to a possible contamination by $[\mathrm{N} \mathrm{II}]$ ). We thus see that a major nebular contribution is implausible for the central component, but cannot be easily excluded in the wings.

Weak 6548.1 and $6583.6 \AA$ [N II] lines are also present in the nebular emission (Harman \& O'Brien 2003; Moraes \& Diaz 2009). There is no evidence in our spectra (e.g. Fig. 3) of a central peak due to these lines, that is, neither for the fainter, $6548.1 \AA$ line nor for the line that is three times stronger at $6583.5 \AA$. The nebular [N II] contribution can thus be deemed undetectible. Separation of the nebular component may be easier in the future with space-resolved spectroscopic observations of the centre of the HR Del image.

With an inclination of $42^{\circ}$, a white dwarf mass of $0.65 M_{\odot}$ and a radial velocity of $250 \mathrm{~km} \mathrm{~s}^{-1}$ (measured from our spectra), the radius of the outermost circular orbit of emission for the central component of the profile would be at $6.2 \times 10^{10} \mathrm{~cm}$ or $0.89 R_{\odot}$ from the centre of the white dwarf stellar component. This is significantly larger than the radius of the photo-ionising disk found from Moraes \& Diaz (2009). The reason for this difference is not clear at present; however, parts of the disk at intermediate radii may not produce much ionising radiation and/or may only contribute to the profile wings. The radius of the emitting region appears somewhat too large when compared with the equatorial radius for an accretion disk around the white dwarf according to the two possible system parameters of Kürster \& Barwig (1988), but the distance from the centre of the white dwarf to the inner Lagrangian point is close to the calculated value of the outer radius of the disk, using the expressions on page 33 of Warner (1995). The simplest explanation of these results is that some radiation is emitted by regions of higher radial velocity at the outer edge of the disk than is directly deduced from the observations, but that it is occulted because of a particular geometry of the line-emitting region.

High-velocity components have been observed in HR Del in the past, in particular in the high velocity C IV resonance line P Cygni absorption component in the ultraviolet, which had an edge radial velocity of $-5000 \mathrm{~km} \mathrm{~s}^{-1}$ (Selvelli \& Friedjung 2003). Such a component could have been produced in a highvelocity jet. No evidence for it is seen today in our $\mathrm{H} \alpha$ profiles

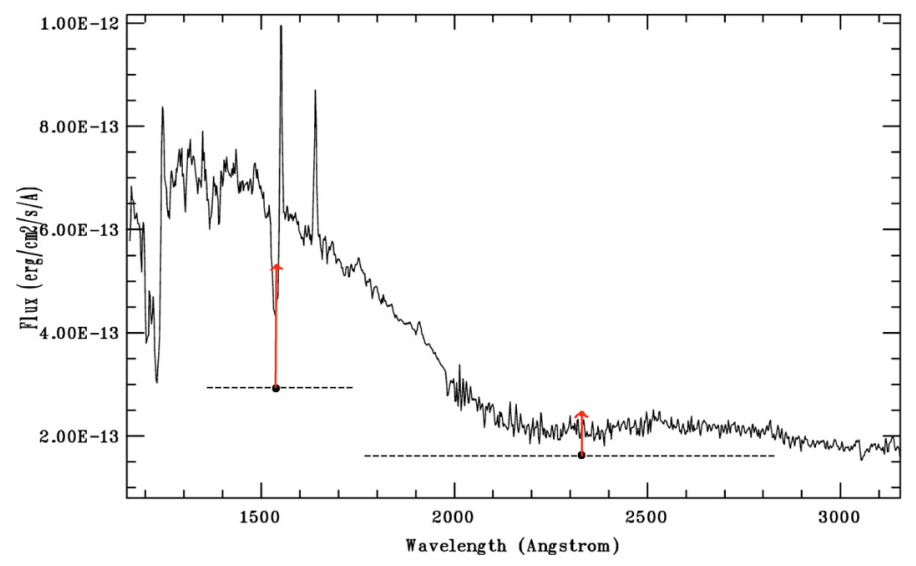

Fig. 14. The two Galex photometric points (lower, black points) superposed on the IUE average spectrum from Selvelli \& Friedjung (2003). The horizontal dashed lines represent the bandpass of the two (NUV and FUV) filters. The upper, red points, linked to the corresponding observed (lower) values, represent the real, estimated flux after correction for deadtime effects in the detectors.

nor in older optical profiles such as in the 1990 spectrum (Ringwald et al. 1996). One can thus wonder to what extent the ultraviolet spectrum of HR Del has changed between the lifetime of IUE and the epoch of our optical spectra, but no UV spectra could be obtained since the end of IUE in 1996. The only recent UV data available are two photometric points from the Galex all-sky survey, which give the following AB magnitudes (observations of 2006 September): 12.78 in the near-UV (central wavelength $2315 \AA$ ) and 12.96 in the far-UV $(\lambda 1539 \AA)$. If taken at face value, these magnitudes would indicate a small fading in the near-UV, and a stronger one (about a factor of two) in the far-UV with respect to the average IUE spectrum from Selvelli \& Friedjung (2003). As however HR Del is slightly brighter than the saturation limit of the Galex detectors, those magnitudes have to be corrected for dead-time effects. When this is done according to the precepts described in Morrissey et al. (2007), the corrected AB magnitudes become 12.36 and 12.38 in the NUV and FUV, respectively.

This means that the NUV magnitude is still about the same as in 1988, within the uncertainties of the correction and that in the FUV the fading has not exceeded $30 \%$ since 1988 , which is quite remarkable. This is illustrated in Fig. 14, which is Fig. 1 of Selvelli \& Friedjung (2003), where we have plotted the Galex values over the spectrum (uncorrected for reddening). Of course, we still need a good UV spectrum to assess what are the real line profiles today.

In connection with non-orbital variations of $\mathrm{H} \alpha$, Selvelli \& Friedjung (2003) suspected quite rapid non-orbital variations of the C IV and N V ultraviolet resonance line absorption in HR Del, similar to the rapid variations of C IV seen for another old nova (V603 Aql in Friedjung et al. 1997, and in Prinja et al. 2000). Such variations in the ultraviolet resonance absorption components of P Cygni profiles may be expected for the winds of accretion disks of cataclysmic binaries and have been observed (Froning 2005; Proga 2005).

The "S wave type" pattern of $\mathrm{H} \alpha$, displayed in Figs. 10 to 12, is quite clear on the blue side, but somewhat less so on the red side of the profiles. It does, however, not appear to be a classical cataclysmic binary $\mathrm{S}$ wave. However, since it moves over the central component of the line profile like the classical wave, it can be understood as near the outer edge of a rotating disk in the neighbourhood of a fixed point in the rotating frame of the 
binary. The profile shows a maximum assymmetry towards the violet around Kürster and Barwig phase 0.75 or at photometric phase 0.33 , which is only 0.08 of an orbital period after photometric phase 0.25 . If we interpret the photometic variations as due to varying visibility of the regions of the surface of the mass loser, heated by radiation from the disk and white dwarf (see below), the photometric zero phase is when the mass loser is nearest the observer in inferior conjunction. The phase of maximum assymetry of the profile around photometric phase 0.33 is then not far from quadrature when the spot is near a line perpendicular to the line of sight. An accretion disk should rotate in the same direction as the binary (conservation of angular momentum), so the $\mathrm{H} \alpha$ bright spot should be on the side of the disk opposite to the mass loser. Such a situation is not classical. That could possibly be related to the large size of the accretion disk found above.

We can emphasise however that the stability of the $\mathrm{S}$ wave over the six years of our spectroscopic observations (about 10000 orbital cycles) indicates that it moves with a period which is quite close to the orbital period of Kürster and Barwig. Indeed, using their estimated error for the period, we calculate that the phase shift over the time of our spectroscopic observations would be of the order of 0.23 . Positive and negative superhump periods, which can be permanent, would, on the other hand, differ from the orbital period by a factor of a few percent when the orbital period is that of HR Del (Olech et al. 2009).

The variations in the $V$ magnitude cannot be caused by the aspect changes of the distorted Roch lobe-filling cool component in a normal state. The visual magnitude was around 12.5 during our observations, which gives an absolute magnitude around 2.35, using a distance of $850 \mathrm{pc}$ and a visual extinction of $A v=0.5$ (Selvelli \& Friedjung 2003). An ordinary star not heated by its companion, with a mass of 0.53 solar masses (mass of the companion, according to Selvelli \& Friedjung 2003) should have an absolute magnitude of the order of 8.5 (assuming the star is on the main sequence). The contribution of an unheated cool component to the $V$ magnitude would thus be around $1 / 300$, and variations in its visibility could not produce the observed photometric changes in $V$ with a full amplitude of $0.08 \mathrm{mag}$. It would moreover be hard to produce the observed symmetrical light curve, by supposing extra light produced by an assymmetrical bright spot.

If the ellipticity (or, more precisely, the shape of the Roche lobe) of the cool component produced the photometric variations, this component would then be strongly heated by the hot component, consisting of a white dwarf plus an accretion disk. As we have seen, this interpretation poses a problem in understanding the phasing of the $\mathrm{H} \alpha$ bright spot. Such an interpretation disagrees with the relative phases of the $S$ wave and the photometric variations unless the $S$ wave is not produced by a classical bright spot. A more detailed discussion of this aspect is beyond the scope of this paper and will be given in a forthcoming one (Voloshina et al., in prep.).

The large "activity" of HR Del found by Selvelli \& Friedjung (2003) and confirmed by the high luminosty determined by Moraes \& Diaz (2009) still appears to be unusual, regardless of the explanation given for it. The high luminosity leads to heating of the mass loser.

Long time-scale variations are apparent both in the total magnitude of the object and in the equivalent width of $\mathrm{H} \alpha$ (see Fig. 15), but the lack of obvious relation between the two does not give us any clues as to their origin. Those long-time scale variations cannot be similar to those of dwarf novae, which, when not in outburst, are fainter than recent old novae and not
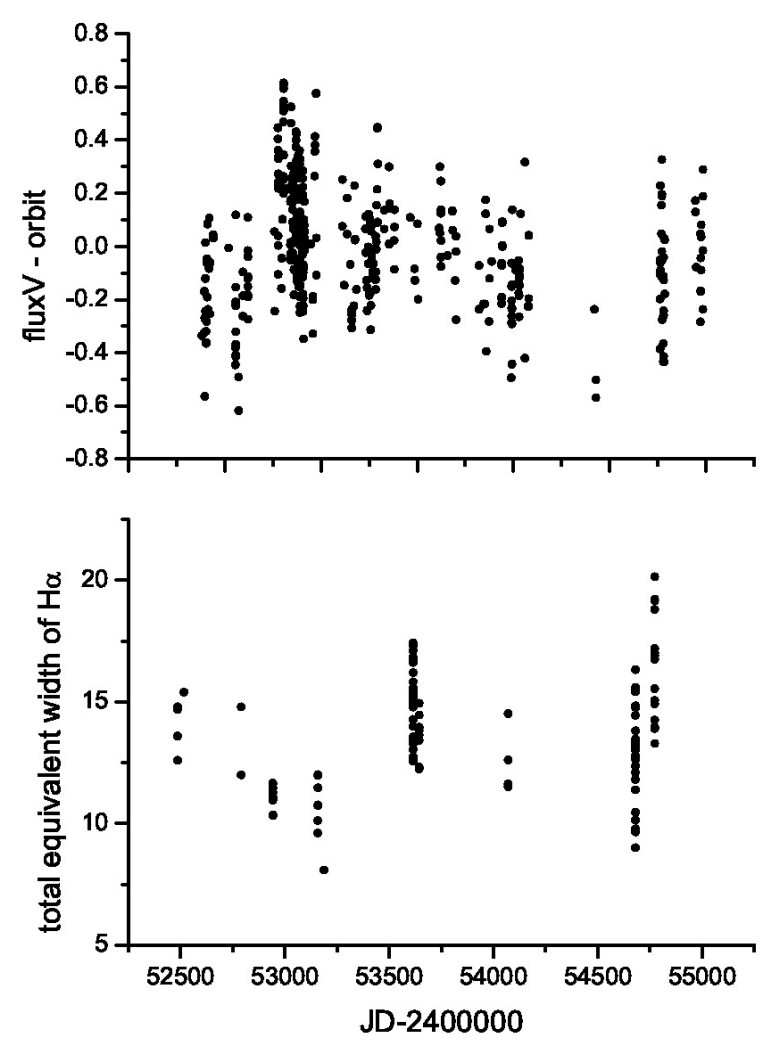

Fig. 15. Combined longer time-scale photometric and spectroscopic behaviour. The upper part shows the $V$ magnitude (corrected for orbital variations) versus Julian date, and the lower part the total equivalent width of $\mathrm{H} \alpha$.

brighter like HR Del. The behaviour of dwarf novae is understood as the result of a lower accretion rate of the white dwarf component outside outburst, compared with that of old novae. Present theories of dwarf nova outbursts explain them by accretion disk instabilities, which can occur at low accretion rates. Small outbursts, somewhat similar to those of dwarf novae, have been observed for the classical novae GK Per and V446 Her some decades after the outburst. This is understood as caused by a drop of the accretion rate at such times, associated with the appearence of instability. The situation is less clear for the lowamplitude (less than $1 \mathrm{mag}$ ), "stunted" outbursts seen for other old novae and novalike systems (Honeycutt et al. 1998).

Solar type cycles of the mass loser, leading to variations in the accretion rate on time scales of about a decade, have also been suggested as an explanation of long-term changes in the luminosity of cataclysmic variables (Bianchini 1987; Warner 1988). It remains to be seen whether this is possible when there is strong irradiation of the mass loser, if one wishes to explain the long timescale variations of HR Del in this way.

\section{Conclusions}

We have performed photometric and high-resolution $\mathrm{H} \alpha$ spectroscopic observations of HR Del from 2002 to 2008. The photometry shows variations depending on orbital phase, plus apparently random variations from night to night and perhaps variations on a longer-time scale of several years. The orbital phase-dependent variations may be interpreted by irradiation of the mass losing companion star by a still very bright, expanded white dwarf and its accretion disk. It is not clear at present 
whether the long time scale variations are interpretable in terms of solar type cycles of the mass loser.

Ultraviolet, broad-band photometric data from the GALEX archive suggest only a weak fading since the epochs of the IUE, but no recent spectra are available to estimate the present activity from the UV spectral lines.

The $\mathrm{H} \alpha$ profile shows both a narrow and a wide component. The former appears to be due to emission by an accretion disk, while the latter probably contains a non-negligible contribution from the expanding nebula. The central component does not show a very clear dependence of equivalent width with orbital phase. Its profile, however, shows a clear orbital variation, which looks similar to the $\mathrm{S}$ wave generally seen in spectra of cataclysmic binaries, but which has the wrong phase dependence to be produced by a classical bright spot. The stability of the $\mathrm{S}$ wave with respect to the orbital period over 8 years indicates no connection with the period of any positive or negative superhump. The exact nature of our "S wave" still remains to be elucidated.

In view of the exceptional brightness and activity of this old nova about 35 years after its eruption, continuing low-level thermo-nuclear combustion in the envelope of a low-mass white dwarf may be considered a possible explanation. The evolution of this interesting stellar system should therefore be closely followed over a large wavelength range in the future.

Acknowledgements. I. Voloshina acknowledges support from the CNRSEARA, which sponsored her visits to the IAP, where part of this work was completed. M. Dennefeld and M. Friedjung thank D. Briot who kindly cooperated in obtaining a long series of spectra in August 2008, and the OHP night assistants (most notably R. Giraud and J.C. Mevolhon) for their valuable contribution to the service observations. The authors are grateful to an anonymous referee for useful suggestions.
Galex is a NASA Small Explorer, developed in cooperation with the French space agency CNES and the Korean Ministry of Science and Technology.

\section{References}

Baldwin, J. A., Philipps, M. M., \& Terlevich, R. 1981, PASP, 93, 5

Bianchini, A. 1987, Mem. Soc. Astr. It., 58, 245

Candy, M. P. 1967, IAU Circ., 2022

Deeming, T. J. 1975, Ap\&SS, 36, 137

Friedjung, M. 1992, A\&A, 262, 487

Friedjung, M., Selvelli, P., \& Cassatella, A. 1997, A\&A, 318, 204

Friedjung, M., Bruch, A., Voloshina, I., et al. 2005, in The Astrophysics of Cataclysmic Variables and Related Objects, ed. J. M. Hameury, \& J. P. Lasota, ASP Conf. Ser., 330, 437

Froning, C. S. 2005, in The Astrophysics of Cataclysmic Variables and Related Objects, ed. J. M. Hameury, \& J. P., Lasota, ASP Conf. Ser., 330, 81

Gillet, D., Burnage, R., Kohler, D., et al. 1994, A\&AS, 108, 181

Harman, D. J., \& O'Brien, T. J. 2003, MNRAS, 344, 1219

Honeycutt, R. K., Robertson, J. W., \& Turner, G. W. 1998, AJ, 115, 2527

Hutchings, J. B. 1980, PASP, 92, 458

Kürster, M., \& Barwig, H. 1988, A\&A, 199, 201

Lamers, H. J. G. L. M., Snow, T. P., \& Lindholm, D. M. 1995, ApJ, 455, 269

Lenz, P., \& Breger, M. 2004, IAUS, 224, 786

Moraes, M., \& Diaz, M. 2009, AJ, 138, 1541

Morrissey, M. P., Conrow, T., Barlow, T. A., et al. 2007, ApJS, 173, 682

Ness, J. U., Schwarz, G., Starrfiels, S., et al. 2008, ApJ, 135, 1228

Olech, A., Rutowski, A., \& Schwarzenberg-Czerny, A. 2009, MNRAS, 399, 465

Prinja, R. K., Knigge, C., Ringwald, F. A., \& Wade, R. A. 2000, MNRAS, 318, 368

Proga, D. 2005, in The Astrophysics of Cataclysmic Variables and Related Objects, ed. J. M. Hameury, \& J. P. Lasota, ASP Conf. Ser., 330, 103

Ringwald, F. A., Naylor, T., \& Mukai, K. 1996, MNRAS, 281, 192

Sanyal, A. 1974, ApJS, 28, 115

Selvelli, P. L., \& Friedjung, M. 2003, A\&A, 401, 297

Sion, E. M., \& Starrfield, S. G. 1994, ApJ, 421, 261

Walraven, Th., \& Walraven, J. H. 1972, in Auxiliary Instrumentation for Large

Telescope, ESO/CERN conference, ed. S. Laustsen, \& A. Reiz, 175

Warner, B. 1988, Nature, 336, 129

Warner, B. 1995, Cataclysmic Variable Stars (Cambridge University Press)

Pages 10 to 11 are available in the electronic edition of the journal at http: //www . aanda. org 
A\&A 521, A84 (2010)

Table 1. Journal of the CCD observations.

\begin{tabular}{|c|c|c|c|c|}
\hline Date & $\begin{array}{l}\text { Set start-end } \\
24000000+\end{array}$ & Bands & Exposure & $N$ \\
\hline 25.08 .2005 & $53608.511-53608.586$ & $\bar{V}$ & $30 \mathrm{~s}$ & 135 \\
\hline 26.08.2005 & $53609.558-53609.582$ & $V$ & $40 \mathrm{~s}$ & 39 \\
\hline 27.08 .2005 & $53610.554-53610.578$ & $V$ & $50 \mathrm{~s}$ & 35 \\
\hline 30.08 .2005 & $53613.541-53613.571$ & $V$ & $60 \mathrm{~s}$ & 32 \\
\hline 31.08 .2005 & $53614.341-53614.371$ & $V$ & $30 \mathrm{~s}$ & 61 \\
\hline 01.10 .2005 & $53645.259-53645.325$ & $V$ & $40 \mathrm{~s}$ & 64 \\
\hline 03.10 .2005 & $53647.234-53647.280$ & $V$ & $40 \mathrm{~s}$ & 80 \\
\hline 13.10 .2005 & $53657.304-53657.342$ & $V$ & $30 \mathrm{~s}$ & 36 \\
\hline 26.07.2006 & $53943.527-53943.594$ & $V$ & $40 \mathrm{~s}$ & 121 \\
\hline 27.07.2006 & $53944.542-53944.592$ & $V$ & $40 \mathrm{~s}$ & 84 \\
\hline 02.08 .2006 & $53950.538-53950.598$ & $V$ & $40 \mathrm{~s}$ & 93 \\
\hline 03.08 .2006 & $53951.557-53951.604$ & $V$ & $40 \mathrm{~s}$ & 73 \\
\hline 04.08 .2006 & $53952.527-53952.602$ & $V$ & $40 \mathrm{~s}$ & 121 \\
\hline 06.08 .2006 & $53954.508-53954.584$ & V & $40 \mathrm{~s}$ & 113 \\
\hline 26.09.2006 & 54005.224-54005.494 & $V$ & $30 \mathrm{~s}$ & 615 \\
\hline 27.09.2006 & $54006.216-54006.463$ & $V$ & $30 \mathrm{~s}$ & 561 \\
\hline 28.09.2006 & $54007.417-54007.473$ & $V$ & $30 \mathrm{~s}$ & 130 \\
\hline 14.10.2006 & $54023.172-54023.408$ & $V$ & $40 \mathrm{~s}$ & 527 \\
\hline 18.10.2006 & $54027.313-54027.430$ & $V$ & $40 \mathrm{~s}$ & 193 \\
\hline 19.10.2006 & $54028.314-54028.428$ & V & $40 \mathrm{~s}$ & 192 \\
\hline 20.10.2006 & $54029.320-54029.406$ & V & $40 \mathrm{~s}$ & 182 \\
\hline 21.10 .2006 & 54030.319-54030.411 & $V$ & $40 \mathrm{~s}$ & 161 \\
\hline 22.10 .2008 & $54762.191-54762.402$ & $V, R$ & $60 \mathrm{~s}, 60 \mathrm{~s}$ & 116 \\
\hline 23.10 .2008 & $54763.182-54763.371$ & $V, R$ & $60 s, 60 s$ & 84 \\
\hline 03.11 .2008 & $54774.158-54774.351$ & $V, R$ & $80 \mathrm{~s}, 80 \mathrm{~s}$ & 104 \\
\hline 04.11 .2008 & $54775.194-54775.267$ & $V, R$ & $80 \mathrm{~s}, 80 \mathrm{~s}$ & 33 \\
\hline 07.11 .2008 & $54778.149-54778.338$ & $V, R$ & $60 \mathrm{~s}, 80 \mathrm{~s}$ & 116 \\
\hline 10.11 .2008 & $54781.216-54781.380$ & $R$ & $200 \mathrm{~s}$ & 50 \\
\hline 11.11.2008 & $54782.166-54782.374$ & $R$ & $240 \mathrm{~s}$ & 83 \\
\hline 12.11 .2008 & $54783.155-54783.360$ & $R$ & $200 \mathrm{~s}$ & 82 \\
\hline 14.11.2008 & $54785.151-54785.282$ & $R$ & $240 \mathrm{~s}$ & 41 \\
\hline 11.10 .2009 & $55116.221-55116.440$ & $V$ & $150 \mathrm{~s}$ & 104 \\
\hline 12.10 .2009 & $55117.185-55117.433$ & $V$ & $150 \mathrm{~s}$ & 117 \\
\hline 13.10 .2009 & 55118.186-55118.318 & $V$ & $150 \mathrm{~s}$ & 64 \\
\hline
\end{tabular}

Table 2. Details of spectroscopic observations.

\begin{tabular}{|c|c|c|c|c|}
\hline Date & $\begin{array}{l}\text { Grating } \\
(1 / \mathrm{mm})\end{array}$ & $\begin{array}{l}\text { UT time of } \\
\text { exposure }\end{array}$ & $\begin{array}{l}\text { Orbital } \\
\text { phases }\end{array}$ & $\begin{array}{c}\text { Equivalent } \\
\text { Width of } \mathrm{H} \alpha \\
\text { Total (Central) }\end{array}$ \\
\hline \multirow[t]{4}{*}{$8 / 8 / 2002$} & 1200 & $00 \mathrm{~h} 50$ & 0.698 & $-13.6(11.6)$ \\
\hline & & $01 \mathrm{~h} 24$ & 0.808 & $-12.6(11.3)$ \\
\hline & & $02 \mathrm{~h} 05$ & 0.941 & $-14.7(13.1)$ \\
\hline & & $02 \mathrm{~h} 38$ & 0.048 & $-14.8(13.2)$ \\
\hline 24/9/2002 & 1200 & $23 \mathrm{~h} 56$ & 0.649 & $-15.4(9.5)$ \\
\hline \multirow[t]{2}{*}{$13 / 6 / 2003$} & 1200 & 01h07 & 0.566 & $-12.0(11.2)$ \\
\hline & & $02 \mathrm{~h} 02$ & 0.744 & $-14.8(13.4)$ \\
\hline \multirow[t]{8}{*}{$1 / 11 / 2003$} & 1200 & $17 \mathrm{~h} 53$ & 0.198 & $-11.7(9.3)$ \\
\hline & & $18 \mathrm{~h} 25$ & 0.302 & $-11.0(8.8)$ \\
\hline & & $18 \mathrm{~h} 58$ & 0.409 & $-10.3(7.3)$ \\
\hline & & $19 \mathrm{~h} 40$ & 0.545 & $-10.3(7.6)$ \\
\hline & & $20 \mathrm{~h} 15$ & 0.659 & $-11.0(7.6)$ \\
\hline & & $20 \mathrm{~h} 55$ & 0.789 & $-11.1(7.3)$ \\
\hline & & $21 \mathrm{~h} 27$ & 0.892 & $-11.3(6.8)$ \\
\hline & & $22 \mathrm{~h} 00$ & 0.999 & $-11.7(8.7)$ \\
\hline \multirow[t]{4}{*}{$2 / 11 / 2003$} & 1200 & $18 \mathrm{~h} 20$ & 0.955 & $-11.5(8.2)$ \\
\hline & & $18 \mathrm{~h} 54$ & 0.066 & $-11.3(8.8)$ \\
\hline & & $19 \mathrm{~h} 40$ & 0.215 & $-10.9(7.6)$ \\
\hline & & 20h16 & 0.331 & $-11.1(7.7)$ \\
\hline \multirow[t]{3}{*}{$6 / 6 / 2004$} & 1200 & 01h12 & 0.859 & $-9.6(7.6)$ \\
\hline & & $01 \mathrm{~h} 42$ & 0.957 & $-10.1(7.8)$ \\
\hline & & $02 \mathrm{~h} 12$ & 0.054 & $-10.8(7.5)$ \\
\hline \multirow[t]{4}{*}{$29 / 6 / 2004$} & 1200 & $00 \mathrm{~h} 37$ & 0.140 & $-12.0(8.8)$ \\
\hline & & 01h07 & 0.237 & $-12.0(8.9)$ \\
\hline & & $01 \mathrm{~h} 40$ & 0.344 & $-11.5(8.2)$ \\
\hline & & $02 \mathrm{~h} 11$ & 0.445 & $-10.7(7.4)$ \\
\hline 25/7/2004 & 1200 & $02 \mathrm{~h} 04$ & 0.824 & $-8.1(5.1)$ \\
\hline \multirow[t]{4}{*}{$6 / 10 / 2004$} & 1200 & 19h10 & 0.009 & $-12.9(11.3)$ \\
\hline & & $20 \mathrm{~h} 00$ & 0.171 & $-13.9(10.5)$ \\
\hline & & 20h34 & 0.282 & $-14.9(11.2)$ \\
\hline & & 21h09 & 0.395 & $-14.0(10.7)$ \\
\hline \multirow[t]{2}{*}{$10 / 9 / 2005$} & 1800 & $22 \mathrm{~h} 07$ & 0.475 & $-12.7(12.1)$ \\
\hline & & $22 \mathrm{~h} 44$ & 0.595 & $-12.6(11.8)$ \\
\hline \multirow[t]{4}{*}{$11 / 9 / 2005$} & 1800 & 20h19 & 0.794 & $-14.3(9.9)$ \\
\hline & & $21 \mathrm{~h} 20$ & 0.992 & $-13.6(9.9)$ \\
\hline & & $22 \mathrm{~h} 20$ & 0.186 & $-15.2(10.3)$ \\
\hline & & $23 \mathrm{~h} 21$ & 0.384 & $-13.0(9.0)$ \\
\hline $12 / 9 / 2005$ & 1800 & 00h33 & 0.617 & $-12.8(8.8)$ \\
\hline \multirow{8}{*}{$13 / 9 / 2005$} & 600 & 20h16 & 0.123 & $-15.5(12.2)$ \\
\hline & & $20 \mathrm{~h} 47$ & 0.223 & $-15.8(12.0)$ \\
\hline & & 21h19 & 0.327 & $-14.0(10.2)$ \\
\hline & & $21 \mathrm{~h} 47$ & 0.418 & $-14.8(10.1)$ \\
\hline & & $22 \mathrm{~h} 17$ & 0.515 & $-14.3(10.1)$ \\
\hline & & $22 \mathrm{~h} 48$ & 0.616 & $-15.6(11.2)$ \\
\hline & & $23 \mathrm{~h} 18$ & 0.713 & $-15.4(11.3)$ \\
\hline & & $23 \mathrm{~h} 48$ & 0.810 & $-13.3(9.2)$ \\
\hline $14 / 9 / 2005$ & 600 & 00h18 & 0.907 & $-17.4(12.6)$ \\
\hline \multirow[t]{6}{*}{$26 / 9 / 2005$} & 600 & $20 \mathrm{~h} 32$ & 0.875 & $-15.3(10.9 .6)$ \\
\hline & & $21 \mathrm{~h} 06$ & 0.986 & $-16.8(12.8)$ \\
\hline & & $21 \mathrm{~h} 36$ & 0.083 & $-16.6(12.9)$ \\
\hline & & $22 \mathrm{~h} 06$ & 0.180 & $-17.1(12.9)$ \\
\hline & & $22 \mathrm{~h} 37$ & 0.281 & $-16.2(12.6)$ \\
\hline & & $23 \mathrm{~h} 08$ & 0.381 & $-13.5(9.4)$ \\
\hline \multirow[t]{4}{*}{$27 / 9 / 2005$} & 600 & $21 \mathrm{~h} 20$ & 0.700 & $-14.9(10.8)$ \\
\hline & & $21 \mathrm{~h} 51$ & 0.801 & $-15.0(11.6)$ \\
\hline & & $22 \mathrm{~h} 23$ & 0.905 & $-16.7(12.8)$ \\
\hline & & $22 \mathrm{~h} 54$ & 0.005 & $-17.3(12.1)$ \\
\hline
\end{tabular}


M. Friedjung et al.: The old nova HR Del

Table 3. Details of spectroscopic observations, continued.

\begin{tabular}{|c|c|c|c|c|}
\hline \multirow[t]{3}{*}{$24 / 10 / 2005$} & 600 & $18 \mathrm{~h} 24$ & 0.201 & $-13.4(10.4)$ \\
\hline & & $18 \mathrm{~h} 55$ & 0.301 & $-14.9(11.4)$ \\
\hline & & $19 \mathrm{~h} 26$ & 0.402 & $-13.7(9.6)$ \\
\hline \multirow[t]{7}{*}{$26 / 10 / 2005$} & 600 & $18 \mathrm{~h} 12$ & 0.500 & $-12.3(10.0)$ \\
\hline & & $18 \mathrm{~h} 42$ & 0.598 & $-12.3(9.8)$ \\
\hline & & 19h12 & 0.695 & $-13.4(10.7)$ \\
\hline & & $19 \mathrm{~h} 42$ & 0.792 & $-13.8(11.3)$ \\
\hline & & $20 \mathrm{~h} 10$ & 0.883 & $-14.5(10.7)$ \\
\hline & & $20 \mathrm{~h} 44$ & 0.993 & $-13.9(9.3)$ \\
\hline & & $21 \mathrm{~h} 07$ & 0.068 & $-14.0(9.8)$ \\
\hline \multirow[t]{4}{*}{$14 / 12 / 2006$} & 1200 & $17 \mathrm{~h} 48$ & 0.512 & $-11.5(9.2)$ \\
\hline & & $18 \mathrm{~h} 22$ & 0.622 & $-12.6(9.6)$ \\
\hline & & $19 \mathrm{~h} 33$ & 0.852 & $-11.6(8.6)$ \\
\hline & & $20 \mathrm{~h} 10$ & 0.972 & $-14.5(9.9)$ \\
\hline \multirow[t]{5}{*}{$26 / 8 / 2008$} & 1200 & $21 \mathrm{~h} 13$ & 0.810 & $-9.0(6.9)$ \\
\hline & & $21 \mathrm{~h} 47$ & 0.920 & $-10.1(8.0)$ \\
\hline & & $22 \mathrm{~h} 18$ & 0.020 & $-11.4(8.2)$ \\
\hline & & $23 \mathrm{~h} 00$ & 0.157 & $-11.0(8.7)$ \\
\hline & & $23 \mathrm{~h} 31$ & 0.257 & $-10.5(8.0)$ \\
\hline \multirow[t]{7}{*}{$27 / 8 / 2008$} & 1200 & 00h03 & 0.361 & $-9.7(7.1)$ \\
\hline & & $02 \mathrm{~h} 08$ & 0.766 & $-9.8(5.4)$ \\
\hline & & $21 \mathrm{~h} 22$ & 0.508 & $-13.2(11.2)$ \\
\hline & & $21 \mathrm{~h} 55$ & 0.615 & $-13.5(11.8)$ \\
\hline & & $22 \mathrm{~h} 36$ & 0.748 & $-13.4(11.4)$ \\
\hline & & $23 \mathrm{~h} 09$ & 0.855 & $-14.8(13.1)$ \\
\hline & & $23 \mathrm{~h} 51$ & 0.991 & $-15.6(12.7)$ \\
\hline \multirow[t]{9}{*}{$28 / 8 / 2008$} & 1200 & $00 \mathrm{~h} 22$ & 0.092 & $-14.8(12.3)$ \\
\hline & & 00h59 & 0.212 & $-12.6(9.8)$ \\
\hline & & $01 \mathrm{~h} 31$ & 0.316 & $-13.8(11.7)$ \\
\hline & & $02 \mathrm{~h} 03$ & 0.419 & $-15.4(11.1)$ \\
\hline & & $02 \mathrm{~h} 41$ & 0.543 & $-13.3(10.5)$ \\
\hline & & $21 \mathrm{~h} 51$ & 0.272 & $-14.8(11.0)$ \\
\hline & & $22 \mathrm{~h} 25$ & 0.382 & $-12.8(10.3)$ \\
\hline & & $22 \mathrm{~h} 56$ & 0.482 & $-12.1(9.6)$ \\
\hline & & $23 \mathrm{~h} 35$ & 0.609 & $-13.0(10.0)$ \\
\hline \multirow[t]{4}{*}{$29 / 8 / 2008$} & 1200 & 00h06 & 0.709 & $-12.4(10.1)$ \\
\hline & & 00h36 & 0.807 & $-13.2(10.6)$ \\
\hline & & 01h14 & 0.930 & $-14.4(10.8)$ \\
\hline & & $01 \mathrm{~h} 45$ & 0.030 & $-16.3(10.9)$ \\
\hline \multirow[t]{9}{*}{$20 / 11 / 2008$} & 1200 & $17 \mathrm{~h} 57$ & 0.734 & $-13.9(10.5)$ \\
\hline & & $18 \mathrm{~h} 28$ & 0.834 & $-13.3(9.9)$ \\
\hline & & $19 \mathrm{~h} 00$ & 0.938 & $-16.9(12.5)$ \\
\hline & & $19 \mathrm{~h} 30$ & 0.035 & $-19.2(14.2)$ \\
\hline & & $20 \mathrm{~h} 00$ & 0.133 & $-18.8(13.4)$ \\
\hline & & 20h30 & 0.230 & $-20.2(14.0)$ \\
\hline & & $21 \mathrm{~h} 04$ & 0.340 & $-19.2(12.3)$ \\
\hline & & $21 \mathrm{~h} 35$ & 0.441 & $-16.9(11.4)$ \\
\hline & & $22 \mathrm{~h} 05$ & 0.538 & $-14.3(9.1)$ \\
\hline \multirow[t]{7}{*}{$21 / 11 / 2008$} & 1200 & $18 \mathrm{~h} 48$ & 0.568 & $-15.1(10.3)$ \\
\hline & & $19 \mathrm{~h} 20$ & 0.672 & $-14.0(10.6)$ \\
\hline & & $19 \mathrm{~h} 50$ & 0.769 & $-15.6(10.8)$ \\
\hline & & $20 \mathrm{~h} 21$ & 0.870 & $-17.2(11.7)$ \\
\hline & & $20 \mathrm{~h} 53$ & 0.974 & $-17.0(11.9)$ \\
\hline & & $21 \mathrm{~h} 24$ & 0.074 & $-16.8(10.9)$ \\
\hline & & $21 \mathrm{~h} 58$ & 0.184 & $-14.9(9.0)$ \\
\hline
\end{tabular}

